\title{
A NEW EVALUATION FOR X-RAY ABSORPTION SPECTRA IN THE XANES REGION
}

Hisanobu WAKITA, Toshio YAMAGUCHI, Manabu FUJIWARA, and Seiichi YAMASHITA

Department of Chemistry, Faculty of Science, FUKUOKA University, Nanakuma, Jonan-ku, Fukuoka 814-01, JAPAN

\begin{abstract}
The electronic states of copper(II) ions containing the square-planar tetraaza and macrocyclic copper(II) complexes were measured in solid state by means of $\mathrm{X}$-ray absorption near edge structure (XANES) The peaks in the measured XANES spectra shifted to lower energy side with increasing electron density of a central copper(II) ion. The molecular orbital calculations for the complexes was carried out by the DV-Xa method, and the theoretical XANES spectra were estimated. The chemical shift using XANES spectra was evaluated by this work.
\end{abstract}

Key words XANES, macrocycles, copper (II) complex, DV-Xa,

It is possible to know the nature of the complex molecules and to design the novel complexes and ligands having the superior properties such as macrocyclic copper(II) complexes, if both the electronic state and the local structure of their central metal ions can be determined under the same conditions. At the rising part of the $\mathrm{X}$-ray absorption edge, $\mathrm{X}$-ray absorption spectrum can be affected by the electronic state of the absorption atom in the compounds. In the present report the coorelation between the XANES spectra of the complexes and the electron-donating abilities of the macrocyclic ligands in used complexes is studied. In addition, some peaks in XANES spectra are assigned on the basis of the results of the molecular orbital calculation using the DV$\mathrm{X}_{a}$ method. The compunds in this paper (Fig. 1) have four-coordinated, stable square-planar structure in both crystal and solution.

\section{EXPERIMENTAL}

$\mathrm{X}$-Ray absorption measurements were performed at the BL-7C station of the Synchrotron Radiation(the Photon Factory) in the National Laboratory for High Energy Physics(KEK) $X$-Ray absorption data were collected in the range from ca.9020 eV around copper K-edge $(8981.83 \mathrm{eV})$ The time to collect X-ray photons was $2 \mathrm{~s}$ at each measuring point. Molecular orbital calculation by using the DV-Xa method for the tetraaza macrocyclic copper(II) complexes were performed with an FACOM M-780/10S computer. The reliable crystal parameters of the molecular structures of the copper(II) complexes have not yet been reported. Therefore, their atomic positions of the macrocyclic ligands were estimated from similar structures in reported analogous complexes. For the $\mathrm{Cu}-\mathrm{N}$ bond distances within the copper(II) complexes was used an average value $(1.98 \AA)$ obtained from XAFS analysis for the macrocyclic copper (II) complexes. The point group symmetries are $\mathrm{C}$ for complexes $\mathrm{A}$ and $\mathrm{B}$, and $\mathrm{D}$ for complexes $\mathrm{C}$ and $\mathrm{D}$ (Fig.1) Numerical atomic orbitals taken into account in the calculations are $1 \mathrm{~s}$ to $4 \mathrm{p}$ ortitals for $\mathrm{Cu}$ atom, $1 \mathrm{~s}$ to $2 \mathrm{p}$ orbitals for $\mathrm{C}, \mathrm{N}$, and 0 atoms, and $1 \mathrm{~s}$ orbital for $\mathrm{H}$ atom. The $\mathrm{DV}-\mathrm{Xa}$ calculations were repeated until the difference of charyes became less than 0.01 electron, where the charges were calculated from the Mulliken population analysis. The values of the ionization energies of $2 p$ orbitals for $N$ and $\mathrm{Cu}$ atoms were calculated from the DV-Xa calculation and compared with those measured by XPS measurements. The calculated values agreed well with the observed values. 


\section{RESULTS AND DISCUSSION}

For the tetraazacopper(II) complexes, the reduction potentials of complexes $A$, $C$, and $\mathrm{D}$ are given in Fig. 1 as $\mathrm{r} . \mathrm{p}$. values. These values were obtained in DMF at $25 q$ by the polarographic method with a dropping mercury electrode and a mercury pool as the working and counter electrodes. All values are negative and become to more negative in order of $A>C>D$; these results indicate that the electron donating ability of the ligands to a central copper(II) ion is stronger in order of $A<C<D$. The XANES spectra of all the complexes are also shown in Fig. 1. Three peaks are recognized in all spectra. The energy values of the first peak are almost the same. For the second peak, however, the peak intensities become smaller in order of $A<B<C<D$, and the peak positions of the complexes shift (called the XANES shift in this work) to the lower energy side from A to $D$. The third peak of the complexes also shifts to lower energy from $A$ to $D$ and the peaks become sharper. This tendency corresponds to the result of the electron donating ability of the ligand to its central copper in complexes $A, C$, and $D$. The third peaks in the XANES spectra shift to lower energy region with increasing electron donating ability of the ligands, that is, with increasing the electron density of a central copper(II) ion. To perform more detailed invesitigation for the XANES peaks, molecular orbital calculations of the complex molecules were carried out, and the first, second and third peaks in the XANES spectra were assigned by comparing them with the corresponding calculated XANES spectra. From the results of $\mathrm{DV}-\mathrm{X}_{a}$ calculations of the complexes, some energy levels of calculated molecular orbitals and their atomic orbital components are obtained. To estimate the probabilities of the 1s electron transition by an electronic dipole theory, theoretical XANES spectra could be produced using MO calculations shown in Fig. 1. The peaks in the XANES spectra clearly shift to the lower energy side with increasing electron density of central copper(II) ion. The peaks of the XANES spectra could be assigned to the electron transition mainly from Culs orbital to Cu4p and/or Cu4s orbitals trom comparison with the theoretical spectra. The values of the atomic-charge of the copper atom and of the bond-charge of the $\mathrm{Cu}-\mathrm{N}$ bonds in the complexes are consistent with their peak positions in the XANES spectra. The spectral peaks measured by the fluorescent $X$-ray and ESCA methods for the central copper(II) ion in these complexes did not show any noticeable shift. The XANES spectroscopy is expected to be used as an analytical method for the characterization of the chemical state of the X-ray absorbing atom.
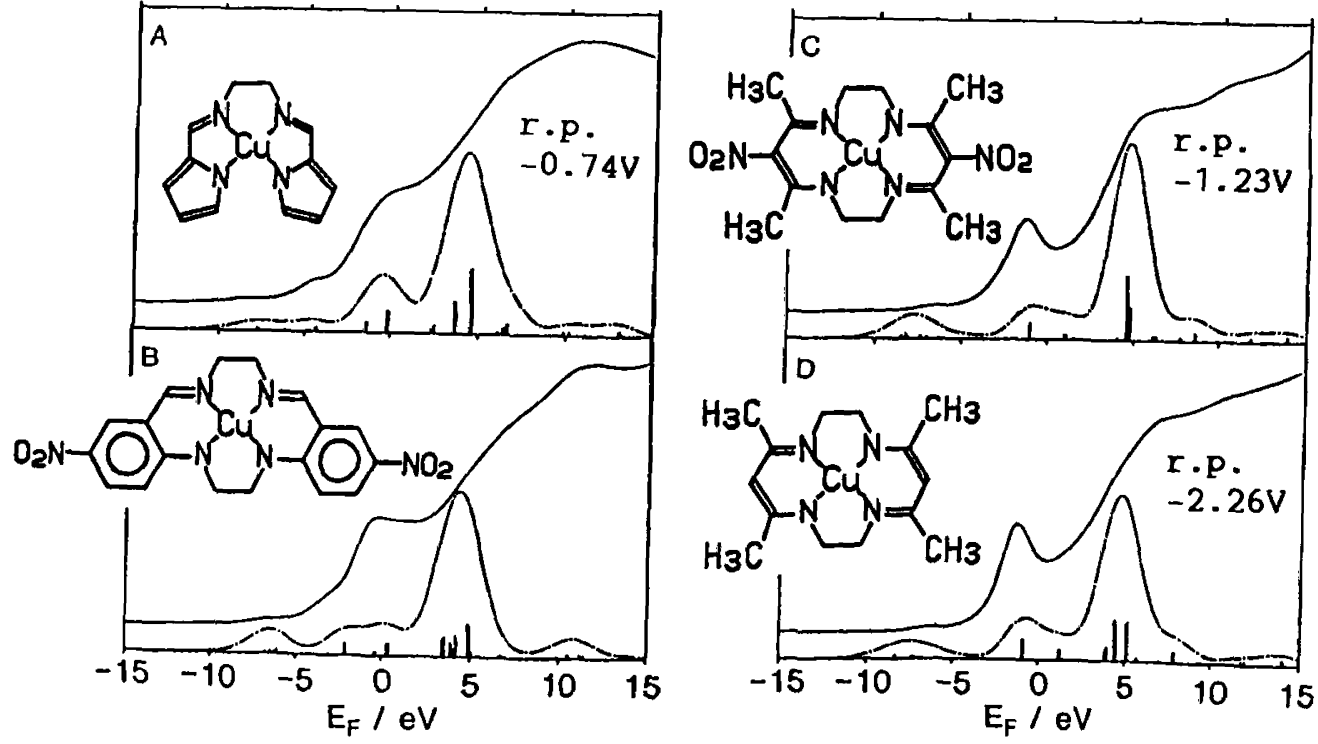

Fig. 1 Theoretical (dashed and dotted line) and measured (solid line) XANES curves of the tetraazacopper(II) complexes. Bar charts represent the calculated transition probability. $E_{F}$ is the relative energy value calculated from the Fermi level. r.p. is the reduction potential value. 